\title{
Analysis of the Influence Mechanism of Energy-Related Carbon Emissions with a Novel Hybrid Support Vector Machine Algorithm in Hebei, China
}

\author{
Jingmin Wang ${ }^{1,2}$, Fan Yang ${ }^{1,2 *}$, Xueting Zhang1, \\ ${ }^{1}$ Department of Economics and Management, North China Electric Power University, Baoding, China \\ ${ }^{2}$ Beijing Key Laboratory of New Energy and Low-Carbon Development \\ (North China Electric Power University), Changping, Beijing, China
}

Received: 4 July 2018

Accepted: 14 August 2018

\begin{abstract}
The Beijing-Tianjin-Hebei Region (BTH) as a national strategic highland attracts attention with its haze problem. In particular, Hebei is a major emitter of carbon emissions in BTH. The establishment of the Xiong'an New District in Hebei, known as the "Millennium plan," faces complex and diverse development in the future, so the carbon emission prediction and influence mechanism are of great significance. This paper has made two improvements to the particle swarm optimization algorithm (PSO), then the improved algorithm is used to optimize parameters of the traditional support vector machine (SVM). Therefore, a new model, IPSO-SVM, is established. This paper uses the STIRPAT model to determine the impact factors, through 64 predict scenarios of 2017-2020 to reveal that economic growth is the most important factor of carbon emissions in Hebei, followed by resident population, industrial structure, urbanization level, energy structure, and technical level. In the case of positive economic development, the contribution of technology to carbon reduction will increase. Based on the "new normal," Hebei ought to develop sustainable urbanization and emphasis on the role of technology in low-carbon development to control carbon emissions.
\end{abstract}

Keywords: influence mechanism; carbon emissions prediction; IPSO-SVM; scenario analysis

\section{Introduction}

China has pledged to the world that the intensity of energy consumption and the intensity of $\mathrm{CO}_{2}$ emissions are used as binding indicators, and that the

*e-mail: bigfan08@163.com carbon intensity of GDP in 2020 will be reduced by $40-45 \%$ compared with 2005 . In order to achieve the early arrival of carbon emission peaks, the Chinese government has formulated another constraint indicator. The 13th Five-Year Plan for controlling greenhouse gas (GHG) emissions requires that the carbon intensity of the Beijing-Tianjin-Hebei region (BTH) in 2020 fall by $20.5 \%$ from 2016. BTH is China's political and cultural center and an important core area of northern China's 
economy, which includes Beijing, Tianjin, and Hebei. China's central government has recently rolled out several national strategies encompassing the BTH, such as "BTH coordinated development" and "Air pollution prevention and control action plan." The carbon emissions in BTH are mainly affected by Hebei. In 2016, the energy consumption of Hebei reached 298.74 million tons of standard coal, which accounted for $6.83 \%$ of the total energy consumption in China, and accounted for $66.51 \%$ in BTH. The CPC Central Committee and the State Council established the Hebei Xiong'an New District, which has been hailed as a "millennium plan." To become an ecological benchmark, new requirements are placed on carbon emissions in Hebei. Meanwhile, Hebei is facing new opportunities, its future development may be complex and diverse, and there are many aspects of studying the significance of carbon emissions. Therefore, when we study the BTH carbon reduction policy, we should focus on Hebei. Urban areas and the rapid progress of industrialization and technology are leading to serious air pollution in urban areas [1-2]. China's urban development inevitably leads to more direct and indirect pollution and has an impact on carbon emissions. In particular, human beings trying to maintain urban life harm health. Population growth and industrialization have led to air pollution in some cities that reaches levels that threaten human health. This has become one of the most important topics of our day. In particular, carbon dioxide $\left(\mathrm{CO}_{2}\right)$ and other pollutants, which provide global warming, have recently attracted attention; because $\mathrm{CO}_{2}$ is one of the most searched gases. Recent studies show PM10, PM2.5 and $\mathrm{CO}_{2}$ air quality indices. There are a lot of studies for carbon emissions, including indoor and outdoor - especially urban cities and parks. They show that PM2.5 affects human health [3-8]. Therefore, it is urgent to study carbon emissions and a control path.

In view of the claims mentioned above, it is necessary to find the main impact factors and future trends of carbon emissions in Hebei. Combining the future strategic positioning has important practical significance for promoting the carbon emission reduction in the entire $\mathrm{BTH}$ and reaching the carbon peak in advance under the new normal. This is also the fundamental method for solving resource bottlenecks and improving the quality of the environment.

Looking at recent literature, there are many ways to predict carbon emissions, which can be divided into two major categories. One is to directly predict carbon emissions based on the decomposition formula, and the other is to establish mathematical models based on influencing factors to predict. The first category is the direct decomposition of carbon influencing factors, and the decomposition method is subdivided into three types: Structure Decomposition Analysis (SDA) [911], Index Decomposition Analysis (IDA) [12-13], and Production Theory Decomposition Analysis (PDA) [14]. Compared to SDA and IDA, PDA requires less data and only needs to summarize panel data [15]. IDA mainly includes Laspeyres decomposition and Divisia decomposition [16], and the LMDI method [17] is a further improvement. Based on LMDI, Moutinho et al. [18] studied the major factors affecting carbon emissions in Europe. Lin and Long [19] adopted the LMDI method to study the methods to promote emission reduction of chemical industry in China. In addition, kaya model is widely used in carbon emission prediction research. The urbanization factor is introduced into the Kaya identity formula to predict the possible carbon emissions of China in 2020 under three different urbanization policy models [20].

In the second category, there are a large number of studies, such as the computable general equilibrium (CGE) model [21]. In addition, the most widely used model is IPTA. Wang and Lin [22] used VAR and STIRPAT models to study the main influencing factors of carbon emissions during the period 1980-2014 in the commercial sector in China. Meng and Niu [23] used logistic functions to simulate carbon emissions based on energy consumption and China's empirical analysis shows that the simulation of Logistic equation is effective. Wang and Ye [24] introduced the exponent of the relevant variables as an exogenous variable into the multivariate grey model in order to quantify the future carbon emissions of China's fossil energy consumption from 2014 to 2020. Ding et al. [25] designed a new optimized grey multivariable model that modifies the background values, to predict China's carbon emissions from fossil fuel combustion in 2014-2020, laying a solid foundation for formulating policies.

However, compared with the above models, due to the simplicity, robustness, suitability for parallel processing, high efficiency and practicality, artificial intelligence technology has been widely used in carbon emission prediction in recent years. Support vector machine (SVM) and least squares vector machines have been proved to be excellent in solving small sample, nonlinear and high dimensional estimation problems. Sun and Liu [26] used LSSVM to predict carbon emissions of China's three major industries, and the final simulation proved the effectiveness of LSSVM. Sun and $\mathrm{Xu}$ [27] analyze factors affecting carbon emissions in Hebei using an improved BP algorithm. Zhao et al. [28] used the Whale Optimization Algorithm (WOA) combined with the LSSVM model to predict that it had a wide range of application prospects. Sulaiman et al. [29] used the Bees algorithm to trace in a deregulated power system, but did not overcome the shortcomings of neural networks, such as premature and overfitting.

On the one hand, most of the current research has innovations in forecasting methods, and to verify the reliability of the methods through past data, but these studies lack prospective analysis of future trends to some extent. On the other hand, existing studies mostly set the scenarios for controlling single-factor variables to predict, and often did not take into account the effects of all factors combined. This paper innovates in the 
following aspects. Firstly, we proposes a new hybrid IPSO-SVM algorithm using non-linear decreasing inertia weight and selective mutation strategy, which can provide a basis for the subsequent carbon emission reduction path. Secondly, we set up 64 scenarios to predict carbon emissions, so we not only determine the key influencing factors and ranks of carbon emission, but also verify the previous views and identify the characteristics of Hebei itself. Thirdly, analyze the mechanism of carbon emissions in 64 scenarios, explore the changes in the contribution of key factors, and find out the differences between scenarios, which helps to analyze the pathways that can reduce carbon emissions in Hebei as soon as possible. Lastly, this paper contributes to a more objective consideration of the various situations of regional development and targeted meaningful urban development proposals.

The remainder of the paper is structured as follows. In Section 2, we calculate the carbon emission of Hebei from 1990 to 2016, and propose the new model that combines improved particle swarm optimization (IPSO) and support vector machine (SVM). Section 3 obtains the influence factors of carbon emissions through the STIRPAT model, and sets up 64 scenarios. Section 4 predicts the results of carbon emissions in base and low-carbon development, and ranks the main influencing factors of Hebei's carbon emissions. Section 5 concludes the analysis results and makes policy recommendations.

\section{Material and Methods}

\section{Data Conversion and Calculation of $\mathrm{CO}_{2}$ Emissions}

The data used in this paper all come from China Energy Statistical Yearbook and Hebei Statistical Yearbook. In order to eliminate the impact of price, GDP and energy intensity are calculated using fixed prices of 2005, which is the base year.

According to IPCC guidelines (IPCC, 2006), carbon emissions can be calculated by calculating each specific type of energy consumption and its corresponding converted standard coal coefficient, and the $\mathrm{CO}_{2}$ emissions conversion coefficient. Thus, the method used to estimate $\mathrm{CO}_{2}$ emissions from 1990-2016 in Hebei is expressed in Eq. (1):

$$
\mathrm{C}=\sum_{i}^{n} C_{i}=\sum_{i}^{n} P_{i} \times S_{i} \times E_{i}
$$

...where $\mathrm{C}$ is the annual total amount of $\mathrm{CO}_{2}$ emissions; $i$ denotes 9 energy categories, including coal, coke, crude oil, gasoline, kerosene, diesel oil, fuel, natural gas and electricity. $C_{i}$ is $\mathrm{CO}_{2}$ emission of the $i^{\text {th }}$ energy; $P_{i}$ is the total final usage of the $i^{t h}$ energy, $S_{i}$ is standard coal conversion coefficient of the $i^{t h}$ energy; $E_{i}$ is the $\mathrm{CO}_{2}$ emission conversion coefficient of the $i^{\text {th }}$ energy. Therefore, the $\mathrm{CO}_{2}$ emissions calculated by Eq. (1) from 2000 to 2016 in Hebei are shown in Fig. 1.

\section{Improved Particle Swarm Optimization Algorithm}

Particle swarm optimization (PSO) is an evolutionary computation technology. It comes from a study of the behavior of birds hunting. The advantage is that it is simple and easy to implement and does not have many parameters to adjust. In this paper, we have made two improvements to the PSO algorithm.

(1) One is to introduce the selective mutation strategy in the genetic algorithm (GA) into the PSO algorithm, that is, to reinitialize certain variables with a certain probability. The mutation operation expands the population search space that is continuously narrowed in the iterations, enabling the particles to jump out of the previously searched optimal value positions, conducting searches in a larger space while maintaining the diversity of the population and improving the possibility of finding better values.

(2) The second improvement is the addition of nonlinear inertia weights. Shi.Y and Eberhart [30] first

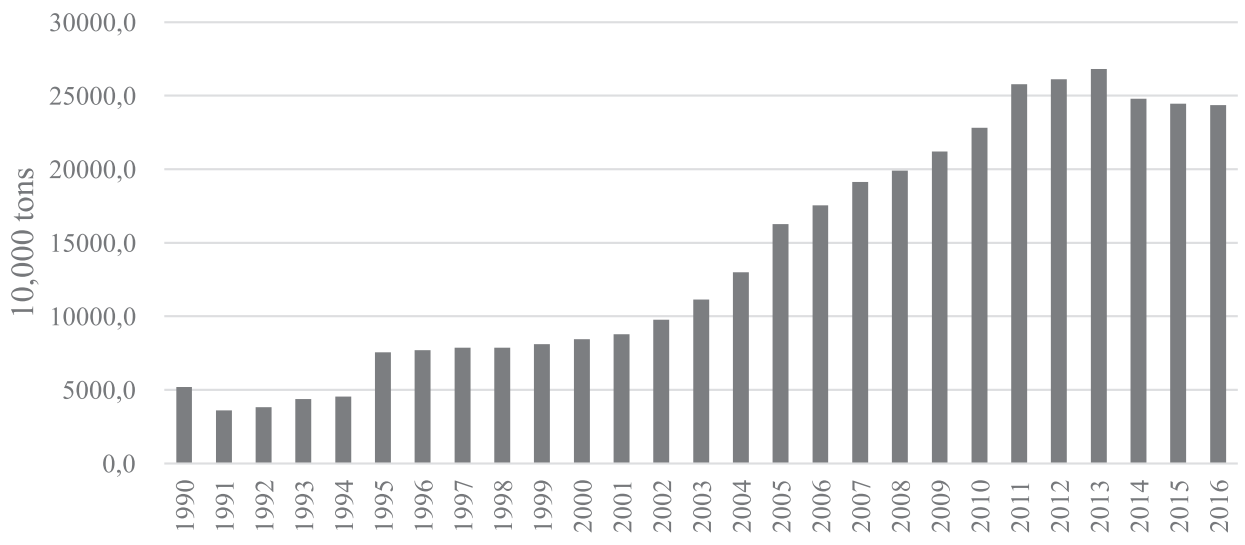

Fig. 1. $\mathrm{CO}_{2}$ emissions in Hebei from 1990 to 2016. 
introduced the inertia weights into the PSO algorithm, and pointed out that a larger inertia weight favors a global search, while a smaller inertia weight is more helpful with a local search. In order to better balance the algorithm's global search and local search ability, this study uses nonlinear decreasing inertia weights (NLDW) [31].

The mathematical model of the PSO algorithm is: assume that in a D-dimensional search space, the particle population consists of $N$ particles; the $i_{\text {th }}$ particle is represented by $X_{i}=\left(X_{i 1}, X_{i 2}, \ldots, X_{i D}\right)$ and it also represents the search space. According to the objective function, the corresponding fitness value of each particle position $X_{i}$ can be calculated.

The $i_{\text {th }}$ particle velocity is expressed as $V_{i}=\left(V_{i 1}, V_{i 2}, \ldots, V_{i D}\right)$; the individual extremum is $P_{i}=\left(P_{i 1}, P_{i 2}, \ldots, P_{i D}\right)$; the population global extremum is $P_{g}=\left(P_{g 1}, P_{g 2}, \ldots, P_{g D}\right)$.

By iterating to find the optimal fitness value, the particles update the speed and position according to Eq. (2):

$V_{i d}^{k+1}=\omega V_{i d}^{k}+c_{1} r_{1}\left(P_{i d}^{k}-X_{i d}^{k}\right)+c_{2} r_{2}\left(P_{g d}^{k}-X_{i d}^{k}\right)$

$$
X_{i d}^{k+1}=X_{i d}^{k}+V_{i d}^{k+1}
$$

The updated equation for the nonlinear decreasing inertia weight coefficient is:

$$
\omega=\left(\omega_{\max }-\omega_{\min }\right) \times\left(\frac{l_{\max }-l}{l_{\max }}\right)^{p}+\omega_{\min }
$$

The NLDW is neither a constant nor a linear gradient, but it is non-linearly decreasing with the number of iterations, speed, and position.

Where $\omega$ is the inertia weight, $r_{1}$ and $r_{2}$ are the random numbers distributed uniformly from 0 to 1 ; $c_{1}$ and $c_{2}$ are acceleration constants. $l_{\max }$ represents the maximum number of iterations; $p$ represents a nonlinear modulation index, equal to 1.2 .

\section{A Novel Hybrid Support Vector Machine Algorithm based on IPSO}

Support Vector Machine (SVM) was originated by Cortes and Vapnik (1995) [32]. As a kind of machine learning algorithm, SVM has outstanding performance in solving high-dimensional, nonlinear, and small sample problems. However, SVM still has disadvantages such as being vulnerable to local extremes and overfitting. We can see that the prediction accuracy of SVM is highly dependent on parameters, and two improvements can effectively improve the accuracy of the IPSO-SVM algorithm. The IPSO-SVM model calculation steps are shown in Fig. 2.

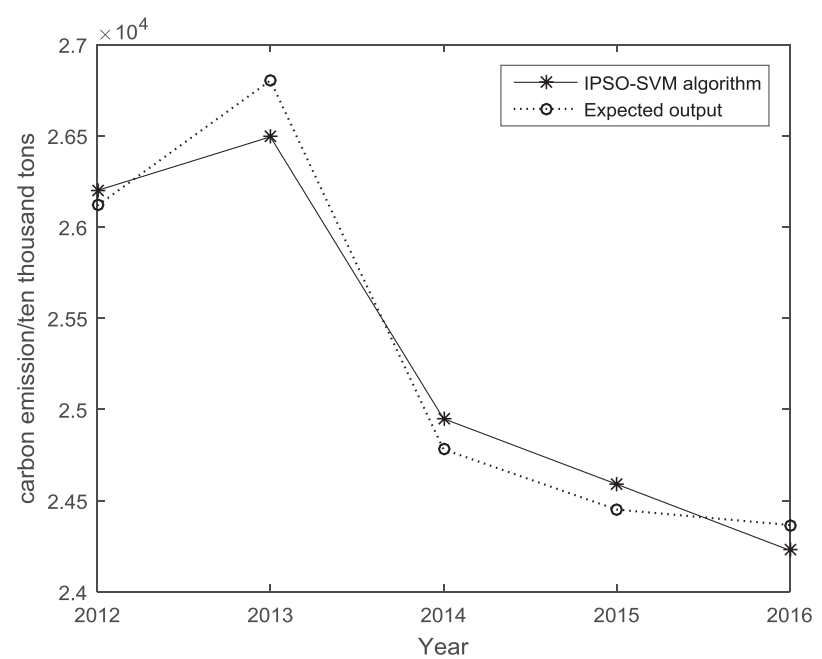

Fig. 2. $\mathrm{CO}_{2}$ emissions prediction of Hebei from 2012 to 2016 .

To verify the accuracy of the improved model, we selected 23 groups of data from 1990 to 2016 in Hebei as the training set for testing, and the remaining 4 sets of data were tested as test sets. The results show that the values of MAPE, MaxAPE, and MdAPE are $0.92 \%$, $1.15 \%$, and $0.41 \%$, respectively. This shows that the model can meet the forecast demand of the future.

$$
\begin{gathered}
\text { Fitness }=M A P E=\frac{1}{n} \sum_{t=1}^{n}\left|\frac{y_{t}-\hat{y}_{t}}{y_{t}}\right| \times 100 \% \\
M a x A P E=\max \left(\left|\frac{y_{t}-\hat{y}_{t}}{y_{t}}\right| \times 100 \%\right) \\
M d A P E=\operatorname{median}_{i}\left(\left|\frac{y_{t}-\hat{y}_{t}}{y_{t}}\right|\right) \times 100 \% \\
M S E=\frac{1}{n} \sum_{t=1}^{n}\left|y_{t}-\hat{y}_{t}\right| \times 100 \%
\end{gathered}
$$

This paper uses the Libsvm3.22 toolbox in Matlab $\mathrm{R} 2015 \mathrm{~b}$ to train SVM based on radial basis function (RBF). The main initial parameters used in the algorithm are shown in Table 1.

Table 1. Parameter setting of IPSO-SVM algorithm.

\begin{tabular}{|c|c|}
\hline Parameters & Setting \\
\hline $\mathrm{c} 1$ & 1.5 \\
\hline $\mathrm{c} 2$ & 1.7 \\
\hline Generations & 300 \\
\hline Population size & 30 \\
\hline Mutation probability & 0.5 \\
\hline Non-linear decreasing inertial weight & 0.9 \\
\hline
\end{tabular}




\section{Results and Discussion}

\section{Influence Factors of $\mathrm{CO}_{2}$ Emission Prediction Model}

IPAT identity is one of the important methods in energy economic research and carbon emission peak prediction. In order to solve the limitations of the independent variables in the IPAT model on the dependent variables, York et al. (2003) [33] established the STIRPAT model on the basis of the IPAT model, whose formula is as follows:

$$
I_{i}=a P_{i}^{b} A_{i}^{C} T_{i}^{d} e_{i}
$$

...where $I$ is the total amount of $\mathrm{CO}_{2}$ emissions; $a$ represents the intercept term; $b, c$, and $d$ are the coefficients of environmental effects with respect to $P$, $A$, and $T ; i$ means the year; and $e_{i}$ means the random error term.

This paper intends to improve three factors in the STIRPAT model. We chose resident population and urbanization level as the P factor, GDP as the A factor, and energy intensity as the $\mathrm{T}$ factor. In addition, we introduced new factors to explain carbon emissions. We chose coal consumption to represent the energy structure, and the proportion of secondary industry to represent industrial structure.
The second step is to apply SPSS 21.0 to conduct binary correlation analysis and significant test and Pearson coefficient calculation on the $\mathrm{CO}_{2}$ emissions preselected factors in Hebei, which are shown in Table 2-4.

\section{Scenario Description}

\section{Economic Growth}

This paper chooses total GDP growth rate to measure economic growth. Combined with the experience of developed countries, when the economy develops rapidly, economic growth will generally tend to be gradual. The $19^{\text {th }}$ National Congress confirmed that China would transform from high-speed growth to high-quality development, which is a strategic change. Under the current "new normal economy," the future economy in Hebei will turn to healthy and green growth. The undertake non-capital function from the Beijing-Tianjin-Hebei integration, the establishment of the Xiong'an New District and the development of the Belt and Road will further stimulate the economic development speed and development quality of Hebei. Therefore, the economic growth rate of Hebei will remain as shown in Table 5.

Table 2. Bivariate correlation.

\begin{tabular}{|c|c|c|c|c|c|}
\hline Factor & $\begin{array}{c}\text { Pearson c } \\
\text { oefficient }\end{array}$ & $\begin{array}{c}\text { Significant } \\
\text { (bilateral) }\end{array}$ & Factor & $\begin{array}{c}\text { Pearson } \\
\text { coefficient }\end{array}$ & $\begin{array}{c}\text { Significant } \\
\text { (bilateral) }\end{array}$ \\
\hline GDP & $0.957^{* *}$ & 0.000 & Coal consumption & $0.999^{* *}$ & 0.000 \\
\hline Resident population & $0.966^{* *}$ & 0.000 & Proportion of secondary industry & $0.715^{* *}$ & 0.000 \\
\hline Urbanization level & $0.968^{* *}$ & 0.000 & Energy intensity & $-0.838^{* *}$ & 0.000 \\
\hline
\end{tabular}

Note: $* *$ indicates a significant correlation at the bilateral significance level of 0.01 .

Table 3. Model summary.

\begin{tabular}{|c|c|c|c|}
\hline $\mathrm{R}$ & R-squared & Adjusted R-squared & Standard estimated error \\
\hline $1.000^{\mathrm{a}}$ & 0.999 & 0.999 & 281.39712 \\
\hline
\end{tabular}

a. Predictive variables (constants): GDP, resident population, urbanization level, coal consumption, proportion of secondary industry, energy intensity.

Table 4. Anova ${ }^{\mathrm{b}}$.

\begin{tabular}{|c|c|c|c|c|c|}
\hline Model & Quadratic sum & df & Mean square & F & Sig. \\
\hline Regression & 1738161742 & 6 & 289693623.7 & 3658.471 & $.000^{\mathrm{a}}$ \\
\hline Residual & 1583686.788 & 21 & 79184.339 & & \\
\hline Total & 1739745429 & 26 & & & \\
\hline
\end{tabular}

a. Predictive variables (constants): GDP, resident population, urbanization level, coal consumption, proportion of secondary industry, energy intensity.

b. Dependent variable: carbon emissions. 
Table 5. Parameters of economic growth rate.

\begin{tabular}{|c|c|c|}
\hline Year & Medium Growth Rate & Low Growth Rate \\
\hline $2017-2020$ & $7 \%$ & $6.5 \%$ \\
\hline
\end{tabular}

Table 6. Parameters of resident population growth rate.

\begin{tabular}{|c|c|c|}
\hline Year & Medium Growth Rate & Low Growth Rate \\
\hline $2017-2020$ & $0.65 \%$ & $0.55 \%$ \\
\hline
\end{tabular}

\section{Resident Population Growth}

In light of the statistical yearbook of Hebei, since 2011 the annual growth rate of the resident population in Hebei has been around $0.6 \%$, and the annual increase has remained above 400,000. In 2014, BTH was implemented as a national strategy in various fields, Hebei inherited the transfer of Beijing and Tianjin industry, and the entry of labor-intensive enterprises also brings a corresponding population. Since 2016, the implementation of the "two-child" policy has increased the birth rate in Hebei by 1.1 percentage points to $12.42 \%$, and further increased to $13.2 \%$ in 2017 . The establishment of the Xiong'an New District in 2017 will attract a large number of talents. According to the plan, the new district will accommodate 5 million people by 2040 and increase the matching population in the surrounding cities (Baoding City). Therefore, the resident population of Hebei will continue to increase for the foreseeable future. Considering the implications of these policies, the resident population growth rate will remain as shown in Table 6.

\section{Urbanization Level}

The development of urbanization will bring high $\mathrm{CO}_{2}$ emissions due to expansion of building area and the demand for transportation. At the global level, global cities occupy $50 \%$ of the world's population. But the carbon emissions will account for $3 / 4$ of the world's total emissions by 2030 [34]. The National New Urbanization Plan (2014-2020) proposes the citizenization mechanism of the agricultural transfer population, and reforms the development of urbanization. The report of the $19^{\text {th }}$ CPC National Congress pointed out that urban agglomerations should be the main body to construct the pattern of coordinated development of various scale cities, so as to accelerate the urbanization of the agricultural transfer population. There is no doubt that the

Table 7. Parameters of urbanization level growth rate

\begin{tabular}{|c|c|c|}
\hline Year & Medium Growth Rate & Low Growth Rate \\
\hline $2017-2020$ & $5.0 \%$ & $3.7 \%$ \\
\hline
\end{tabular}

Table 8. Parameters of energy structure adjustment rate.

\begin{tabular}{|c|c|c|}
\hline Year & Medium Growth Rate & Low Growth Rate \\
\hline $2017-2020$ & $-2.5 \%$ & $-1.9 \%$ \\
\hline
\end{tabular}

establishment of BTH urban agglomeration has driven the pace of urbanization (Table 7).

\section{Energy Structure}

This paper chooses coal consumption to measure the energy structure. In recent years, the implementation of residents' heating "change coal to gas" (Energy Development Strategic Action Plan, 2014-2020), industrial and commercial clean heating [35], and rail transit development have promoted the elimination of coal in some cities and increased rural scattered coal governance. The efforts will further reduce the consumption of coal. In addition to the full use of electric vehicles, and Hebei is expected to form 500,000 new energy vehicle production capacity by 2020 (the $13^{\text {th }}$ Five-Year Plan for Developing New Energy Vehicle Industry in Hebei Province, 2016-2020) will also increase the clean energy absorption capacity (Table 8).

\section{Industrial Structure}

This paper uses the proportion of secondary industry to measure. The industrial sector is currently the most important contributor to carbon emissions; their share of contributions has never been lower than $40 \%$ since 2001. However, the process of BeijingTianjin-Hebei industrial transfer will lead to a new round of industrial structure optimization in Hebei, and economic development is accelerating the transformation of the dominant form of the tertiary industry, helping to reduce carbon emissions. The establishment of Xiong'an New District will also continue to enhance the upgrade. In 2015, the Central Financial Leading Group Meeting proposed that "supply-side structural reforms" will help improve the quality and efficiency of the supply system, promote the effective elimination of excess production capacity, and promote the optimization and reorganization of industries (Table 9).

Table 9. Parameters of industry structure adjustment rate.

\begin{tabular}{|c|c|c|}
\hline Year & Medium Growth Rate & Low Growth Rate \\
\hline $2017-2020$ & $-2.1 \%$ & $-3.0 \%$ \\
\hline
\end{tabular}

Table 10. Parameters of technical level growth rate.

\begin{tabular}{|c|c|c|}
\hline Year & Medium Growth Rate & Low Growth Rate \\
\hline $2017-2020$ & $-6.0 \%$ & $-4.0 \%$ \\
\hline
\end{tabular}




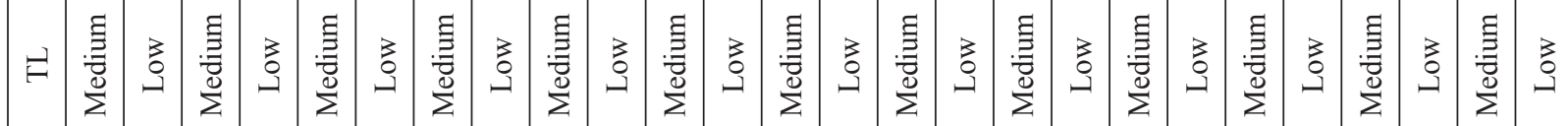

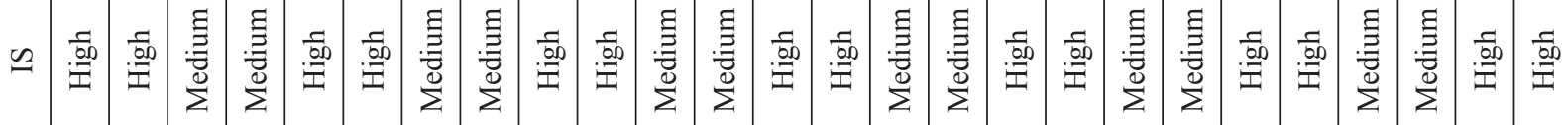

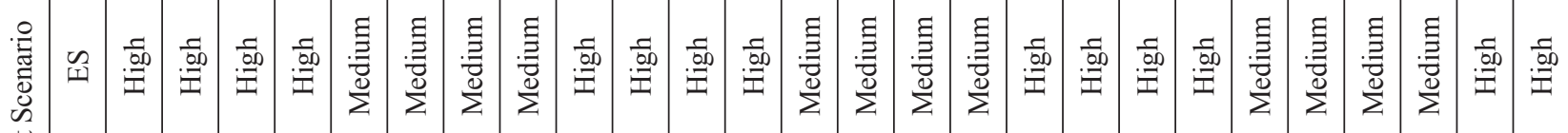

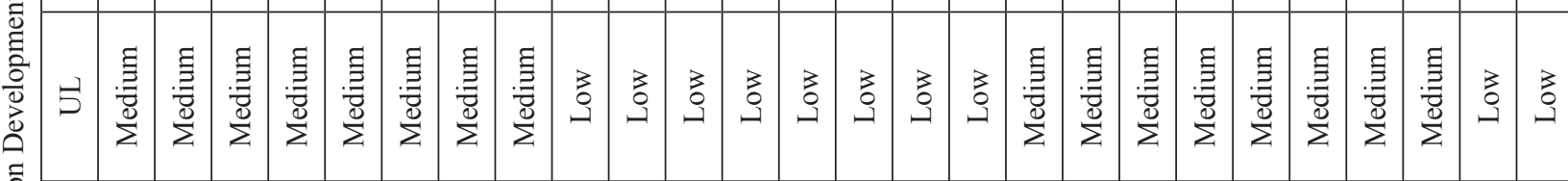

Uू.

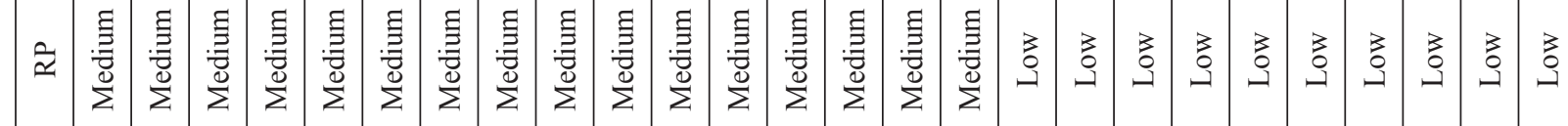

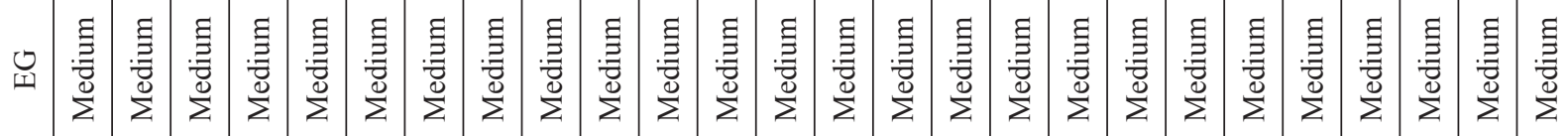

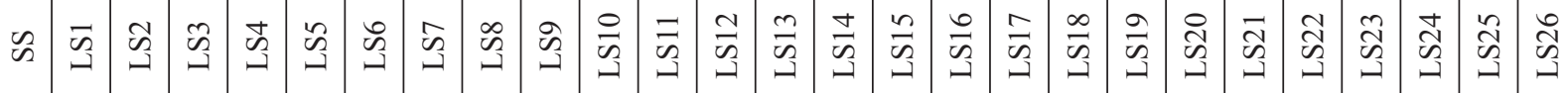

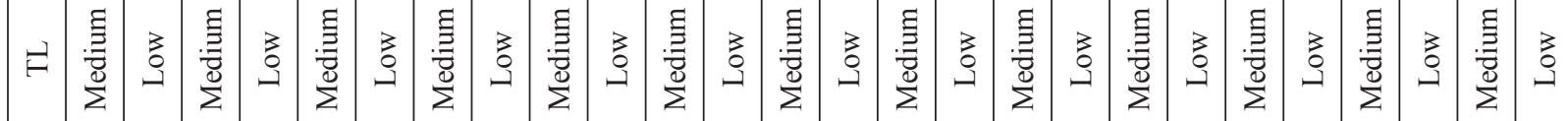

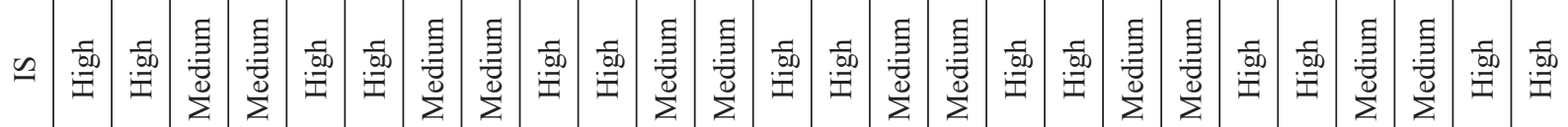

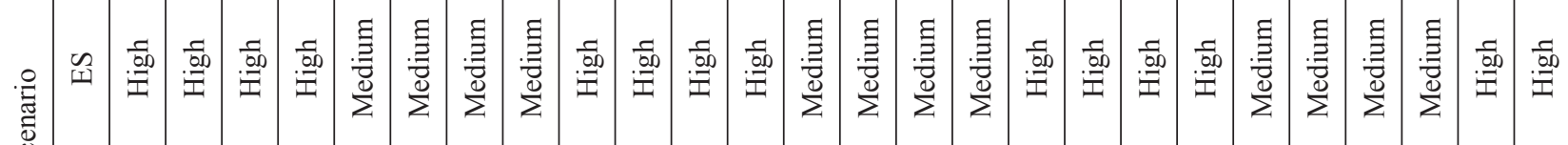

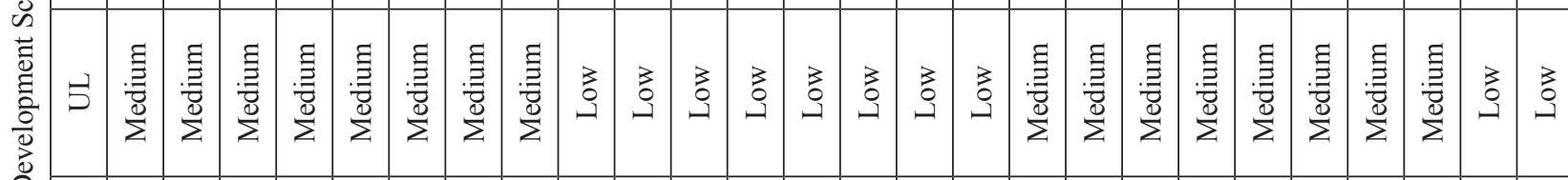

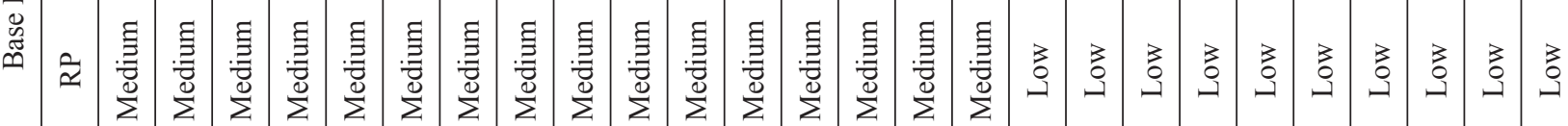

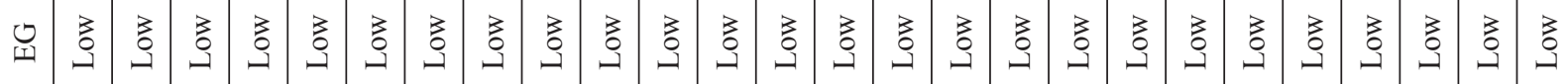

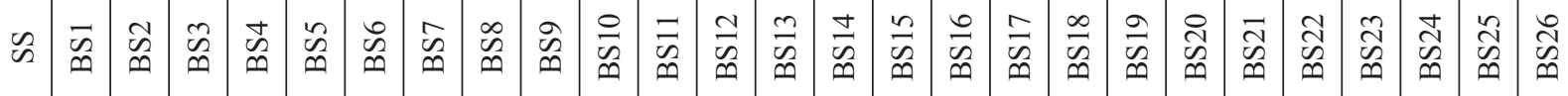




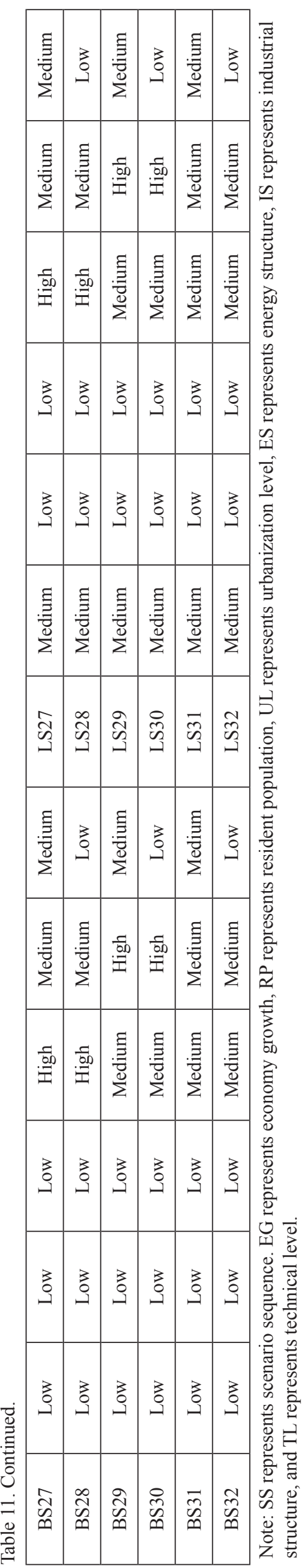

\section{Technical Level}

Energy intensity is used to measure technical level. During 2016 to 2002, the government will keep on vigorously developing efficient production methods and improve energy efficiency [36]. In undertaking the transfer of industries from Beijing and Tianjin, it will also undertake energy-saving equipment and technologies. Also, the transferred talents have correspondingly heightened the management of energy production and consumption. Energy-efficient buildings, electric vehicles and agglomeration effects of emerging industries all increase the level of technology (Table 10).

Therefore, the final scenario includes six factors divided into two categories: base development scenarios and low-carbon development scenarios. A total of 64 scenarios are shown in Table 11.

\section{Prediction Results in the Base Development Scenario}

The carbon emissions prediction results of the 32 base development scenarios are shown in Fig. 3. The economic development rate of these scenarios is set to low speed. From the results, we can see that the overall carbon emission trend in Hebei is increasing. The carbon emissions growth rate in 2020 will fluctuate between $12 \%$ and $15 \%$ relative to 2017 , and the average annual growth rate will be $4.4 \%$. This is in line with Hebei's development status, industrial sector accounts for a relatively large proportion and is still in the period of industrial upgrading. Therefore, in the short term, carbon emissions will continue to increase, and carbon intensity will decline. Carbon emissions will reach the maximum value in scenario BS8, in which the resident population growth rate, urbanization level, industry structure and energy structure adjustment remain the medium rate, while technical progress will remain low. In contrast, carbon emissions will reach the minimum value in scenario BS25, five corresponding factors are at the level of low, low, high, high, medium.

By comparing BS8 with BS7, BS4, BS6, BS16 and BS24, following five carbon emission prediction values are in descending order, and each scenario parameter setting value changes one factor compared with the highest value BS8. From this, we can conclude that when the single-factor effect, the economic growth is at a low speed, the role of the population is the greatest, followed by the industrial structure, urbanization level, energy structure, and technical level. Using the quotient of emission reductions and BS8 carbon emissions to define a factor's contribution (like (BS8-BS7)/BS8), the contribution of these five factors to carbon reduction is $1.90 \%, 1.49 \%, 1.31 \%, 0.55 \%$, and $0.30 \%$, respectively. At the same time, the increase in population and urbanization is a positive factor leading to an increase in $\mathrm{CO}_{2}$, while industrial structure, energy structure adjustment, and technical level can inhibit $\mathrm{CO}_{2}$. 


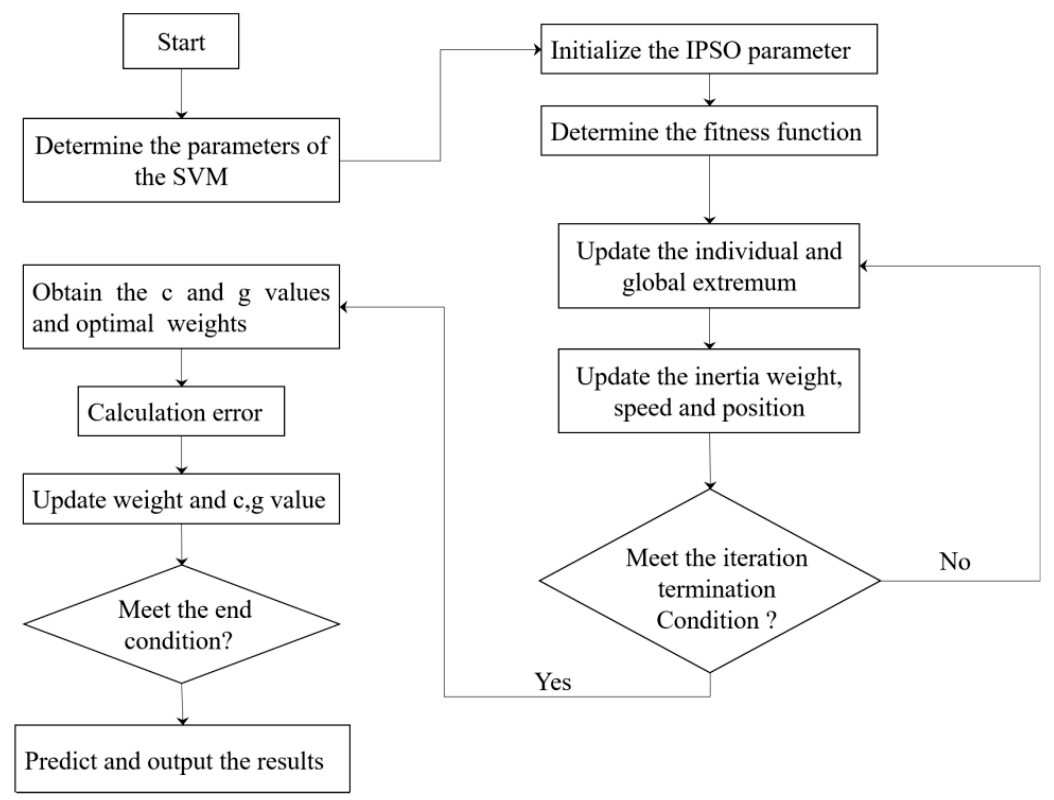

Fig. 3. Flowchart for IPSO-SVM model.

With the GDP growth rate kept at a low speed, the changes in the contribution of five other factors each year are shown in Fig. 4.

This result is in line with the status quo in Hebei. First of all, Hebei has a large population and is the sixth most populous province in the country. The population (especially the urban population) will directly increase the consumption of industry, transportation, and construction, and the latter three are the main contributors to carbon emissions. In 2015, the Chinese government proposed a "two-child policy" that will change the population structure and increase the number of laborers, which may lead to a continued increase in carbon dioxide emissions.

Second, the proportion of secondary industry of Hebei is large, with the policy for the integration of the BTH, Hebei will accelerate the upgrading of industry in a short time. In 2017, the assembly manufacturing industry surpassed the iron and steel industry as the first pillar industry, the service industry contributed more to economic growth than secondary industry, and carbon emissions slowed down during the same period, indicating that the adjustment of industrial structure to carbon reduction is effective.

Urbanization has a strong positive impact on carbon emissions. Urban expansion requires more infrastructure, transportation and personal resource consumption, all of which increase energy consumption. In 2000, the urbanization rate in Hebei is $19.6 \%$, which reached $53.3 \%$ in 2016 . The migration of a large number of people from rural areas to cities has also reduced the proportion of primary industries. In 2016, the government put forward "Enforced Opinions on the Implementation of New Urbanization Construction" and actively facilitated the urbanization of agricultural transfer population. Therefore, due to the acceleration of urbanization, it has gradually become one of the main factors that increase carbon emissions, which is consistent with the findings of Yang et al. [37].

Finally, we can find that the technical contribution to carbon reduction in Hebei is not very high, which is related to the long cycle of technology from development to introduction and popularization, such as energy auditing and energy efficiency supervision, which all

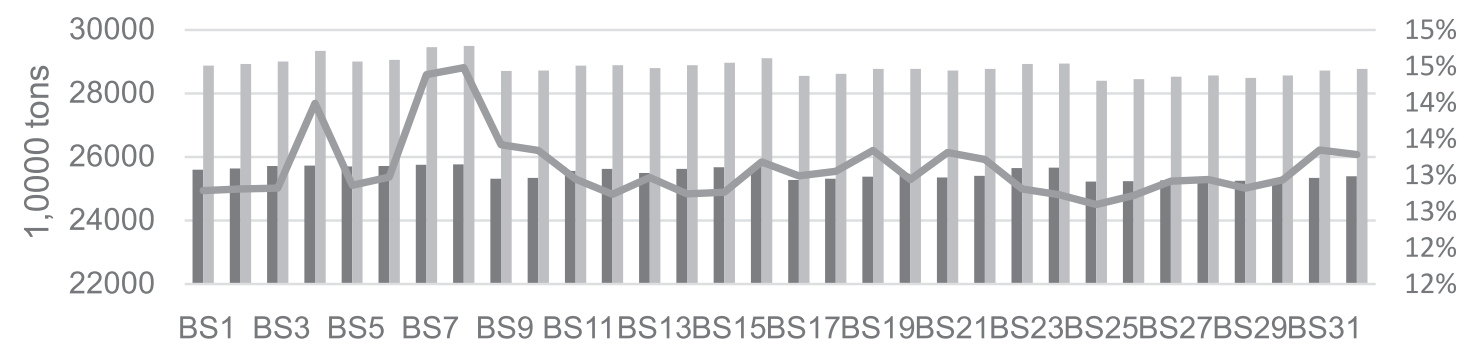

Fig. 4. Flowchart for IPSO-SVM model. 


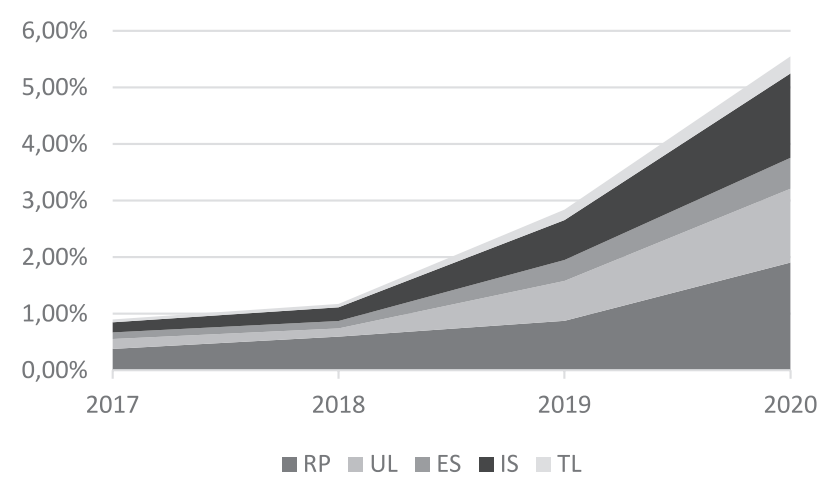

Fig. 5. Contributions of 5 factors in the base development scenario.

take a relatively long time to accumulate, so as to have higher contribution, so the contribution of technical level in the short term is not very great. From previous research, the conclusion is different from Wang et al. [38]. He believes that technology is an important factor in four major Chinese cities: Beijing, Tianjin, Shanghai and Guangzhou. This paper deems that the differences in the development stages of the cities have resulted in different levels of technical contribution. Beijing and Tianjin have unique geographical advantages; Shanghai and Guangdong are located in the urban agglomeration named the Yangtze River Delta and Pearl River Delta that were developed earlier in China. Earlier introduction of advanced technologies and the exchange of energy-saving and emission-reduction technologies with foreign countries are more frequent in these cities. Hebei is located in China's inland, where the BeijingTianjin-Hebei urban agglomeration started late and the level of city development is still at an immature stage, resulting in the current technology being less important for carbon reduction. This coincides with the view of Shuai et al. [39], that the higher the income, the greater the technical impact on carbon emissions.

By comparing BS3 and BS5, BS4 and BS6, BS11 and BS13, BS14 and BS16, BS19 and BS21, BS22 and BS24, BS29 and BS31, and BS28 and BS30, we can further see that the industrial structure and energy structure have different effects on carbon emission inhibition. The former has an inhibitory effect 1.36 times that of the latter. Similarly, comparing BS1 and BS19, BS2 and BS20, BS5 and BS21, BS6 and BS22, BS9 and BS27, BS10 and BS28, BS10 and BS28, BS14 and BS30, we can find that the effect of reducing the population growth rate on the suppression of carbon emissions is obvious, which is 1.41 times that of industrial restructuring.

\section{Prediction Results in the Low-Carbon Development Scenario}

The predicted $\mathrm{CO}_{2}$ of 32 low-carbon scenarios in Hebei during 2017-2020 are shown in Fig. 5. The economic development of these scenarios is set to medium-speed, with an annual growth rate of $7.0 \%$. Compared with the base scenario, the growth rate of $\mathrm{CO}_{2}$ has dropped significantly. The average growth rate fluctuates between $4 \%$ and $7 \%$, and the average value is close to $2.0 \%$. Carbon emissions will reach their peak in scenario LS8, in which the population, urbanization level, industry structure and energy structure adjustment rate are at the medium level, while technical remains low. In contrast, carbon emissions will reach the minimum value in scenario LS25, in which five corresponding factors are at the level of low, low, high, high, medium. Through the comparison between LS8 and LS7, LS4, LS6, LS16, LS24, it is still concluded that the role of population is the most important in the single factor effect, followed by industrial structure, urbanization level, energy structure, and technical level. The effect of the five factors of reducing emissions was $1.45 \%, 0.92 \%, 0.91 \%, 0.45 \%$, and $0.44 \%$, respectively. From the prediction results of low-carbon scenarios, we can see that the speed of economic development has slightly increased by $0.5 \%$, the overall importance of the remaining five factors will not be changed, but it will affect the contribution of each factor.

By comparing LS3 and LS5, LS4 and LS6, LS11 and LS13, LS14 and LS16, LS19 and LS21, LS22 and LS24, LS29 and LS31, LS28 and LS30, it is further seen that Industrial structure and energy structure have different impacts on carbon emission inhibition once again. The former has an inhibitory effect 1.38 times that of the latter. Similarly, comparing LS1 and LS19, LS2 and LS20, LS5 and LS21, LS6 and LS22, LS9 and LS27, LS10 and LS28, LS10 and LSS28, LS14 and LS30, we

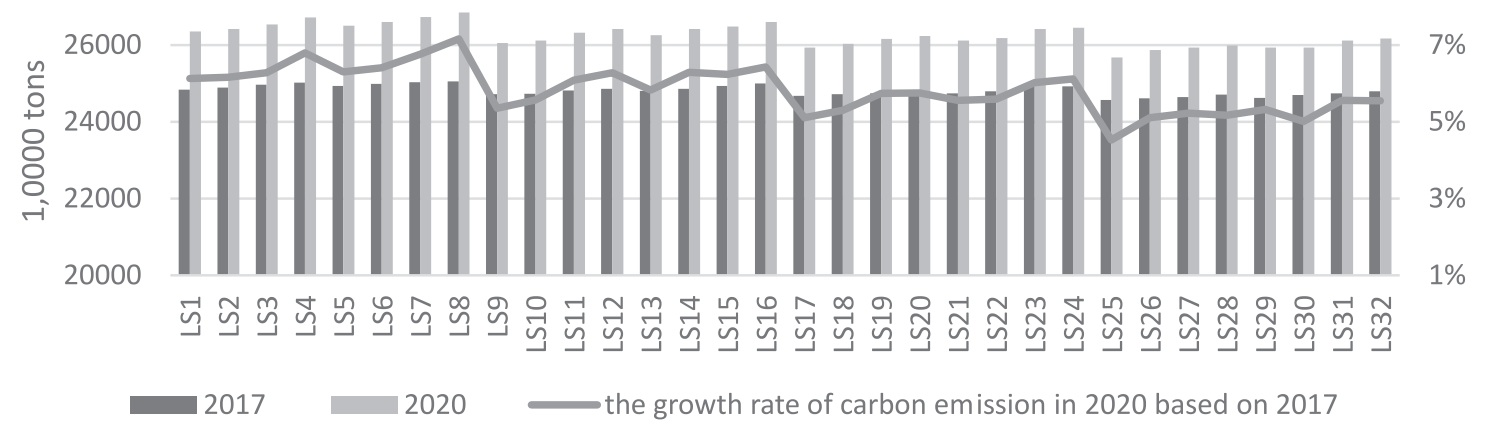

Fig. 6. 32 scenarios of $\mathrm{CO}_{2}$ emission prediction of Hebei in the low-carbon development scenario. 


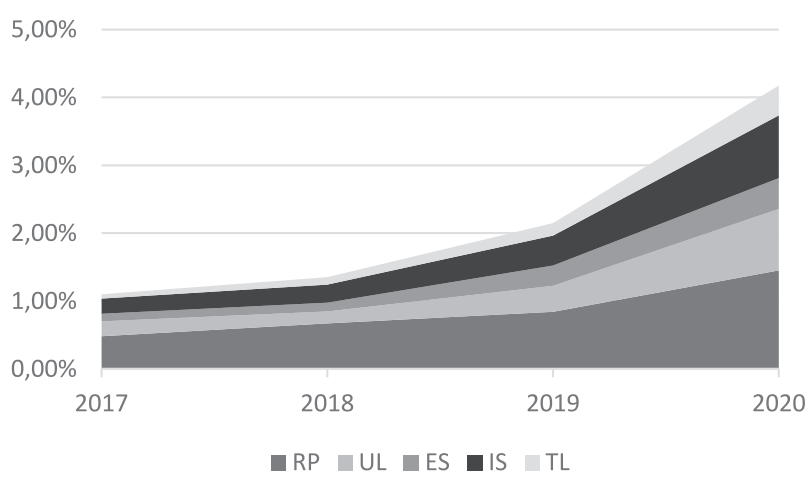

Fig. 7. Contributions of 5 factors in the low-carbon development scenario.

can see that the effect of reducing the population growth rate to suppress carbon emissions is more pronounced, which is 1.72 times that of industrial restructuring.

With the GDP growth rate kept at a medium speed, the changes in the contribution of five other factors each year are shown in Fig. 6.

\section{Comparison of Base Development Scenario and Low Carbon Scenario}

By comparing the carbon emissions of above two major scenarios, we can find that the economic factor is the most paramount in the decline of $\mathrm{CO}_{2}$, which verifies the view of Shuai et al. [40]. In the base scenario, the growth rate of economic indicators is $6.5 \%$, and the annual average increase rate of carbon emissions in Hebei is $4.4 \%$; the two ratios in low carbon scenarios are $7.5 \%$ and $2.0 \%$, respectively. This result shows that under the low-carbon development requirements, the economy has gradually decoupled from energy consumption. The path of technical progress is highly related to the economy, agreed to Kang et al. [41], and its contribution rate has increased from $0.30 \%$ to $0.44 \%$. It shows that the more economically developed, more funds can be introduced to develop new technologies, use energy-saving machines, which can reduce carbon emissions fundamentally. The Beijing-Tianjin-Hebei

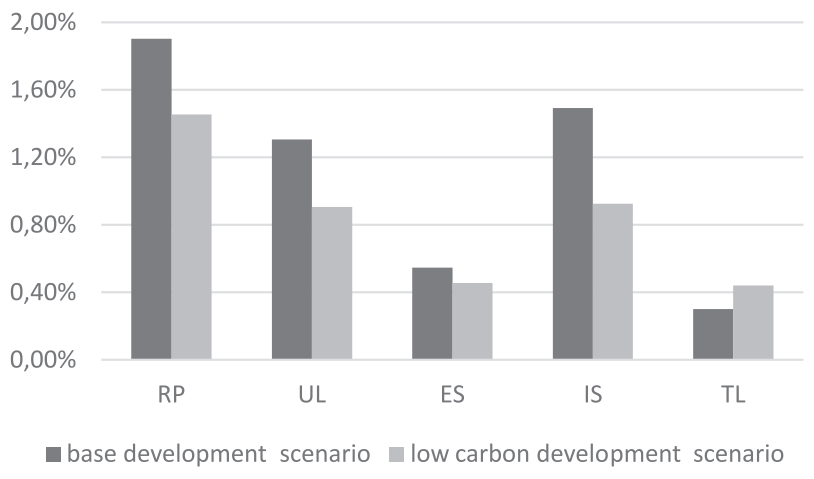

Fig. 8. Comparison of contributions of main factors in two kinds of scenarios. region, as an emerging urban agglomeration, currently has strong policy support. Hebei will continue to inject large amounts of funds and human capital from the two mega cities, Beijing and Tianjin, and will have a relatively impressive economic growth rate in the future. The direct effect is to improve energy efficiency, and the indirect effect is to promote the progress of the high-tech industry, optimize the industrial structure, and jointly reduce carbon emissions. Therefore, economic development can increase the inhibitory effect of technology on carbon emissions. At the same time, Fig. 7 illustrates that the speed of the industrial structure adjustment is smaller than technical level improvement; however, the total contribution of the industrial structure is higher than that of technology. This indicates that the existing "industrial structure bias" in Hebei is the main problem.

Comparing the 64 scenes, the importance of industrial restructuring is greater than the adjustment of energy structure. It might be the fact that their average contribution values are $0.65 \%$ and $0.25 \%$, respectively. It is related to the industrial upgrading and optimization efforts of Hebei in recent years. In 2017, the equipment manufacturing industry surpassed the iron and steel industry both in terms of scale and growth, as well as the proportion of industry and growth, and become the province's first pillar industry. The gradual transition from a heavy industry that relies extremely on energy consumption to a technologyoriented industry will greatly reduce the consumption of coal. Therefore, there is a causal relationship between industrial restructuring and energy structure adjustment. To sum up all the conclusions, economics, population, industrial structure, urbanization level, energy structure, and technology are important factors affecting carbon emissions.

\section{Conclusions}

This paper applies inertial weight and selective mutation strategy to improve PSO. Then the parameters of traditional SVM are optimized with IPSO. We introduce the new model of IPSO-SVM to predict the carbon emissions of different scenarios in Hebei from 2017 to 2020. Comparing 64 kinds of different development scenarios, the main findings can be summarized as follows: (1) The rank of key impact factors of carbon emissions in Hebei are economic growth, resident population, industrial structure, urbanization level, energy structure and technical level. Population and urbanization have a positive effect on $\mathrm{CO}_{2}$ emissions, industrial structure, energy structure, and technology have inhibitory effects on it. (2) Economic growth is the most important factor for carbon emissions in Hebei, and it can change the contribution of other factors. (3) The level of technology can play a greater role in the period of rapid economic development. The better the economic development, 
the greater contribution to carbon reduction the technology will be. Based on this, we propose the following suggestions.

(1) Taking into account Hebei's unique location advantage in the BTH and the establishment of the Xiong'an New District within its territory, the economy will continue to develop at a relatively high speed in the future. Therefore, economic development that does not overly rely on energy consumption becomes the path of carbon reduction. According to the analysis, "insufficient emerging industries" and "emphasis on industrial structure" are the shortcomings of Hebei's economic development. In the future, it is necessary to combine the industrial transfer of Beijing and Tianjin, appropriately reduce the proportion of the secondary industry, vigorously develop tertiary industry, and focus on the development of advanced equipment manufacturing, "Dazhi Moving Cloud," a new generation of information industry and other seven major emerging industrial clusters.

(2) The effect of population and urbanization level on carbon emissions is positive. Because the positive effect of urbanization on environment in the long term is stronger than the short-term impact, in the long run, the level of urbanization develops to a certain level (generally considered as more than $80 \%$ ) will inhibit the growth of carbon emissions. Therefore, the strategy of sustainable development should be reflected in the process of promoting urbanization. We can adopt a government-led approach to establish a carbon-reducing incentive-restriction mechanism so as to enhance the environmental protection consciousness of urban residents and entrepreneurs. Also, government should continue to provide support for the rural population in urban areas.

(3) Policy makers should show solicitude for the gradually increasing role of technology and strengthen human capital to promote imitation innovation. In the early stage of low-carbon development, $R \& D$ investment should be intensified on the basis of active absorption and introduction of technology; in the middle period of development, the leading role of independent innovation in carbon reduction should be emphasized.

(4) Hebei forms an energy structure dominated by coal consumption. Reducing the proportion of coal and optimizing the energy structure should be the focus of emission reduction in the future. Coal is mainly used for power generation and heating, so it is imperative to carry out "clean heating operations in winter" (gas-power generation, district heating, etc.), increase the proportion of renewable energy consumption, and actively form a scientific and rational multi-complementary energy consumption structure.

\section{Acknowledgements}

This paper is supported by the Social Science Foundation of Beijing (Project ID 15JGB050).

\section{Conflict of Interest}

The authors declare no conflict of interest.

\section{References}

1. YAO X., KOU D., SHAO S., LI X., WANG W., ZHANG C. Can urbanization process and carbon emission abatement be harmonious? New evidence from China. Environ Impact Assess Rev, 71, 70, 2018.

2. SHAO L., LI Y., FENG K., MENG J., SHAN Y., GUAN D. Carbon emission imbalances and the structural paths of Chinese regions. Applied Energy, 215, 396, 2018.

3. CETIN M., SEVIK H. Measuring the Impact of Selected Plants on Indoor $\mathrm{CO}_{2}$ Concentrations. Polish Journal of Environmental Studies, 25 (3), 973, 2016.

4. CETIN M., SEVIK H., SAAT A. Indoor Air Quality: the Samples of Safranbolu Bulak Mencilis Cave. Fresenius Environmental Bulletin, 26 (10), 5965, 2017.

5. CETIN M., SEVIK H., ISINKARALAR K. Changes in the particulate matter and $\mathrm{CO}_{2}$ concentrations based on the time and weather conditions: the case of Kastamonu. Oxidation Communications, 40 (1-II), 477, 2017.

6. SEVIK H., CETIN M. Effects of Water Stress on Seed Germination for Select Landscape Plants. Polish Journal of Environmental Studies, 24 (2), 689, 2015.

7. GUNEY K., CETIN M., GUNEY K.B., MELEKOGLU A. The Effects of Some Hormones Applications on Lilium martagon L. Germination and Morpholgical Characters. Polish Journal of Environmental Studies, 26 (6), 1, 2017.

8. TURKYILMAZ A., SEVIK H., CETIN M., AHMAIDA SALEH E.A. Changes in Heavy Metal Accumulation Depending on Traffic Density in Some Landscape Plants. Polish Journal of Environmental Studies, 27 (5), 2277, 2018.

9. WANG H., ANG B., SU B. Multiplicative structural decomposition analysis of energy and emission intensities: Some methodological issues. Energy, 123, 47, 2017.

10. ANG B. LMDI decomposition approach: A guide for implementation. Energy Policy, 86, 233, 2015.

11. WANG Y., ZHAO H., LI L., LIU Z., LIANG S. Carbon dioxide emission drivers for a typical metropolis using input-output structural decomposition analysis. Energy Policy, 58, 312, 2013.

12. ZHAO Y., WANG S., ZHANG Z., LIU Y., AHMAD A. Driving factors of carbon emissions embodied in China - US trade: a structural decomposition analysis. Journal of Cleaner Production, 131, 678, 2016.

13. SHAO S., LIU J., GENG Y., MIAO Z., YANG Y. Uncovering driving factors of carbon emissions from China's mining sector. Applied Energy, 166, 220, 2016.

14. LI A., ZHANG A., ZHOU Y., YAO X. Decomposition analysis of factors affecting carbon dioxide emissions across provinces in China. Journal of Cleaner Production, 141, 1428, 2017.

15. WEINZETTEL J., KOVANDA J. Structural Decomposition Analysis of Raw Material Consumption. J Ind Ecol, 15, 893, 2011.

16. FAN F., LEI Y. Factor analysis of energy-related carbon emissions: a case study of Beijing. J Clean Prod, 163, 277, 2017.

17. LIU L., FAN Y., WU G., WEI Y. Using LMDI method to analyze the change of China's industrial $\mathrm{CO}_{2}$ emissions 
from final fuel use: An empirical analysis. Energy Policy, 35 (11), 5892, 2007.

18. MOUTINHO V., MOREIRA A., SILVA P. The driving forces of change in energy-related $\mathrm{CO}_{2}$ emissions in Eastern, Western, Northern and Southern Europe: The LMDI approach to decomposition analysis. Renewable and Sustainable Energy Reviews, 50, 1485, 2015.

19. LIN B., LONG H. Emissions reduction in China's chemical industry - Based on LMDI. Renewable and Sustainable Energy Reviews, 53, 1348, 2016.

20. WU Y., SHEN J., ZHANG X., SKITMORE M., LU W. The impact of urbanization on carbon emissions in developing countries: a Chinese study based on the U-Kaya method. Journal of Cleaner Production, 135, 589, 2016.

21. AUFFHAMMER M. The rationality of EIA forecasts under symmetric and asymmetric loss. Resource and Energy Economics, 29 (2), 102, 2007.

22. WANG A., LIN B. Assessing $\mathrm{CO}_{2}$ emissions in China's commercial sector: Determinants and reduction strategies. Journal of Cleaner Production, 164, 1542, 2017.

23. MENG M., NIU D. Modeling $\mathrm{CO}_{2}$ emissions from fossil fuel combustion using the logistic equation. Energy, 36(5), 3355, 2011.

24. WANG Z., YE D. Forecasting Chinese carbon emissions from fossil energy consumption using non-linear grey multivariable models. Journal of Cleaner Production, 142, 600, 2017.

25. DING S., DANG Y., LI X., WANG J., ZHAO K. Forecasting Chinese $\mathrm{CO}_{2}$ emissions from fuel combustion using a novel grey multivariable model. Journal of Cleaner Production, 162, 1527, 2017.

26. SUN W., LIU M. Prediction and analysis of the three major industries and residential consumption $\mathrm{CO}_{2}$ emissions based on least squares support vector machine in China. Journal of Cleaner Production, 122, 144, 2016.

27. SUN W., XU Y. Using a back propagation neural network based on improved particle swarm optimization to study the influential factors of carbon dioxide emissions in Hebei Province, China. Journal of Cleaner Production, 112, 1282, 2016.

28. ZHAO H., GUO S., ZHAO H. Energy-Related $\mathrm{CO}_{2}$ Emissions Forecasting Using an Improved LSSVM Model Optimized by Whale Optimization Algorithm. Energies, 10 (7), 874, 2017.

29. SULAIMAN M., MUSTAFA M., SHAREEF H., ABD. KHALID S. An application of artificial bee colony algorithm with least squares support vector machine for real and reactive power tracing in deregulated power system. International Journal of Electrical Power \& Energy Systems, 37 (1), 67, 2012.

30. SHI Y., EBERHART R.C. A modified particle swarm optimizer. Proceedings of Congress on Evolutionary Computation, 69, 1998.

31. CHATTERJEE A., SIARRY P. Nonlinear inertia weight variation for dynamic adaptation in particle swarm optimization. Comput Oper Res, 33 (3), 859, 2006.

32. CORTES C., VAPNIK V. Support-vector networks. Mach Learn, 20 (3), 273, 1995.

33. YORK R., ROSA E., DIETZ T. STIRPAT, IPAT and IMPACT: analytic tools for unpacking the driving forces of environmental impacts. Ecological Economics, 46 (3), $351,2003$.

34. CHEN W., LU Y. The Framework, Routes, and Visions for a Low Carbon City: A Case Study Of Shanghai, Science Press: Beijing, 63, 2010.

35. LIU Z., XU W., ZHAI X., QIAN C., CHEN X. Feasibility and performance study of the hybrid ground-source heat pump system for one office building in Chinese heating dominated areas. Renewable Energy, 101, 1131, 2017.

36. WANG J., YANG F., ZHANG X., ZHOU Q. Barriers and drivers for enterprise energy efficiency: An exploratory study for industrial transfer in the Beijing-Tianjin-Hebei region. Journal of Cleaner Production, 2018. doi:10.1016/j. jclepro.2018.07.327.

37. YANG L., XIA H., ZHANG X., YUAN S. What matters for carbon emissions in regional sectors? A China study of extended STIRPAT model. Journal of Cleaner Production, 180, 595, 2018,

38. WANG S., LIU X., ZHOU C., HU J., OU J. Examining the impacts of socioeconomic factors, urban form, and transportation networks on $\mathrm{CO}_{2}$ emissions in China's megacities. Applied Energy, 185, 189, 2017.

39. SHUAI C., SHEN L., JIAO L., WU Y., TAN Y. Identifying key impact factors on carbon emission: Evidences from panel and time-series data of 125 countries from 1990 to 2011. Applied Energy, 187, 310, 2017.

40. SHUAI C., CHEN X., WU Y., TAN Y., ZHANG Y., SHEN L. Identifying the key impact factors of carbon emission in China: Results from a largely expanded pool of potential impact factors. Journal of Cleaner Production, 175, 612, 2018.

41. KANG Z., LI K., QU J. The path of technological progress for China's low-carbon development: Evidence from three urban agglomerations. Journal of Cleaner Production, 178, 644, 2018. 